\title{
Molecules pressured to react
}

Crystals have been made that undergo reactions when compressed. Computational simulations of these processes provide much-needed atomic-level insight into the mechanisms of mechanically induced reactions. SEE LETTER P.505

STUART L. JAMES

$\mathrm{V}$ arious energy sources can be used to induce chemical reactions - often heat, but also electricity, light and even ultrasound. But there is another option that has been much less explored: mechanical energy from, for example, grinding reactants together or applying pressure to solids. Mechanochemical reactions have been intensively researched in the past few years, but remain poorly understood at the atomic level. On page 505, Yan et al. ${ }^{1}$ provide some much-needed insight into these processes.

Mechanochemistry has a long history and is currently undergoing a renaissance ${ }^{2}$. The ancient Greek philosopher Theophrastus described how cinnabar (mercury sulfide) can be converted into elemental mercury by grinding it in a copper mortar and pestle with vinegar. In the nineteenth century, Michael Faraday studied mechanochemical reactions ${ }^{3}$, and the chemist M. Carey Lea is often credited with demonstrating that mechanically induced reactions can yield different products from thermally induced ones ${ }^{4}$. Polymer chains have also been observed to snap under mechanical stress, and mechanochemical reactions can be expected wherever materials are degraded by mechanical forces. ${ }^{5}$.

Although mechanochemistry was neglected for many years as a viable method for manufacturing chemicals, in the past decade it has attracted much interest in this regard. This is partly because mechanochemical reactions normally need little or even no solvent, and can therefore save on waste and cost compared with conventional, solvent-based synthetic methods ${ }^{6}$. But despite its growing importance, mechanochemistry remains something of an enigma - exactly how is mechanical energy translated into chemical reactivity?

Yan et al. shed some light on this intriguing question. Their work centres on the effect of mechanical force on molecules that consist of carbon-based organic parts connected to an inorganic core of copper and sulfur atoms (Fig. 1). The copper-sulfur core is mechanically 'soft' and susceptible to rupture when a mechanical force is applied; such mechanically sensitive regions of molecules are generally known as mechanophores. By contrast,

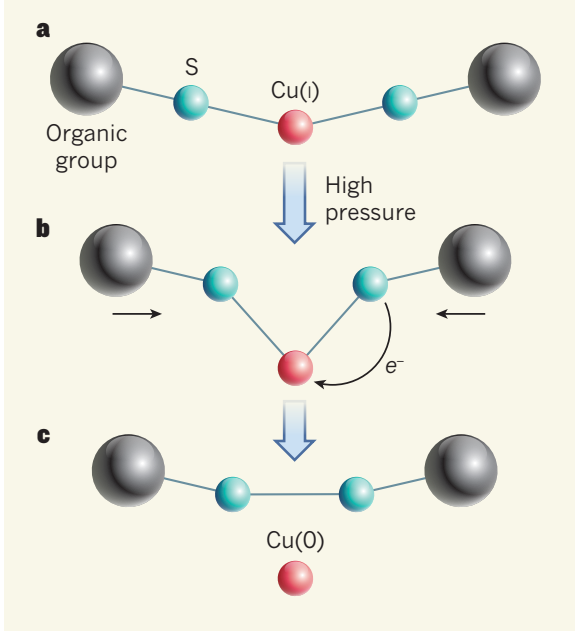

Figure 1 | Reactions induced by compressive force. a, Yan et al. ${ }^{1}$ report molecules that contain incompressible organic groups attached to a 'soft' core consisting of sulfur atoms and copper(I) ions. Only part of the molecule is shown, for simplicity. b, When crystals of the molecules are subjected to high pressure (12 gigapascals or more), the organic groups shift towards each other. This alters the bond angles between the atoms in the core, and induces a redox reaction in which electrons $\left(\mathrm{e}^{-}\right)$move from the sulfur atoms to the copper(I) ions. c, The reaction products are copper $(0)$ (that is, copper metal) and compounds that contain sulfur-sulfur bonds.

the organic parts are mechanically 'hard'.

The authors applied pressure of up to 12 gigapascals to crystals of these molecules and observed a reaction in which nanometre-scale particles of metallic copper were produced, as well as organic by-products that contain sulfur-sulfur bonds. In this mechanochemical reaction, electrons are transferred from sulfur to copper, chemically reducing the copper from copper(I) to metallic cop$\operatorname{per}(0)$. The outcome of the mechanochemical reaction was different from that of heating, which instead produced copper(I) sulfide $\left(\mathrm{Cu}_{2} \mathrm{~S}\right)$ - that is, the mechanochemical and thermochemical reactions broke and formed different chemical bonds. This brings us back to the question of precisely how the mechanochemical process occurs at the atomic level.

Yan and colleagues used computational modelling to show that the application of pressure to the crystals results in 'non-isotropic' compression of the molecules - the mechanically hard organic groups squash the soft copper-sulfur core, dramatically distorting the bond angles between the copper and sulfur atoms. The modelling also showed that, simultaneously with the bond-angle changes, the copper-sulfur bonds become weaker and electron density transfers from the sulfur to the copper atoms. These effects neatly explain the mechanochemical process that leads to the formation of copper-metal particles.

The authors went on to study a variant of their molecule that also contains hard units attached to a soft core but that does not undergo the mechanochemical reaction. Their modelling suggested that the difference in reactivity between the two molecules is due to subtle differences in their structures and crystal packing - for the mechanochemical reaction to occur, the hard organic groups need to have sufficient wiggle room in the crystal to exert force on the copper-sulfur core.

The work is interesting in several further regards. Although a range of mechanophores is known, they are mainly based on organic chemistry and have been explored in the context of polymers. In particular, the rupture of polymer chains caused by stretching can be controlled so that it occurs at specific mechanophore sites ${ }^{5}$. By contrast, Yan and colleagues' mechanophores are inorganic units that undergo redox processes, which occur in response to compression rather than stretching. Moreover, the authors have successfully applied the mechanophore concept to crystals of small molecules, rather than to long-chain polymers. Compressive mechanochemistry such as this might offer a general way to produce nanoparticles.

However, mechanochemical reactions are extremely diverse, and the mechanism put forward in this work helps to explain only a relatively small subset of them. Nevertheless, atomic-level insight into how mechanochemical reactions can occur is invaluable, and Yan and colleagues' work will no doubt stimulate further efforts to reach a similar level of understanding for other reactions, such as those relevant to the synthesis of commercial materials and pharmaceuticals. The authors' 
use of modelling is particularly effective, and gets around the practical difficulties of directly observing mechanochemical reactions at atomic resolution using microscopy.

Finally, the work points to a future in which an informed understanding of mechanochemistry will improve the prediction and design of reactions. This would provide a more rational basis for the field and facilitate the application of mechanochemical reactions as a mainstream synthetic method. .

Stuart L. James is in the School of Chemistry and Chemical Engineering, Queen's University Belfast, Belfast BT9 5GE, UK.

e-mail:s.james@qub.ac.uk
1. Yan, H. et al. Nature 554, 505-510 (2018).

2. Takacs, L. Chem. Soc. Rev. 42, 7649-7659 (2013).

3. Faraday, M. Q. J. Sci. Lit. Arts 8, 374-376 (1820).

4. Lea, M. C. Am. J. Sci. 43, 527-531 (1892).

5. May, P. A. \& Moore, J. S. Chem. Soc. Rev. 42, 7497-7506 (2013).

6. James, S. L. et al. Chem. Soc. Rev. 41, 413-447 (2012).

\section{Force-activated ion channels in close-up}

\section{Piezo proteins allow cells to sense forces by letting ions pass through the cell membrane in response to mechanical stimuli. Three structures of a Piezo protein shed light on how this crucial process works. SEE ARTICLES P.481 \& P.487}

$\mathrm{A}$ 11 cells can sense mechanical force, but it is not clear exactly how. It is known, however, that two closely related members of the mammalian Piezo family of membrane proteins, Piezo1 and Piezo2, have key roles in many physiological and developmental processes that involve mechanosensitivity, including touch, breathing and vascular development ${ }^{1}$. Two papers in Nature, by Saotome et al. ${ }^{2}$ (page 481) and Zhao et al. ${ }^{3}$ (page 487), and a third published in eLife by Guo and MacKinnon ${ }^{4}$, now report structures of Piezol from the mouse (mPiezo1), obtained using a technique known as cryogenic electron microscopy (cryo-EM). The structures provide insight into how Piezo proteins might sense and respond to mechanical force.

It has been difficult to identify mechanically activated channels - proteins that enable a cell to sense force by allowing ions to pass through the cell membrane in response to mechanical stimuli. A major advance in this field was the discovery of the mechanosensitive Piezo channels ${ }^{5}$, which are found in many invertebrates, vertebrates and plants. The mPiezo1 protein is unusually large compared with most proteins, consisting of 2,547 amino-acid residues, and is structurally unrelated to most other proteins.

A previously reported cryo-EM study ${ }^{6}$ provided the first insight into the architecture of mPiezo1. It revealed that the channel is a homotrimer (constructed from three identical subunits) consisting of three 'propeller blades' around a central pore. However, the relatively low resolution of the structure allowed only 14 out of the 38 transmembrane helices in each subunit to be resolved, and none of the amino-acid side chains.

The latest cryo-EM structures ${ }^{2-4}$ have significantly higher resolution (about 4 ångströms) and reveal many crucial structural features of mPiezo1. The channel is a triskelion, with each arm (or propeller blade) consisting of nine repeated structural units, each containing four transmembrane $a$-helices. These arms surround a central pore formed by the two transmembrane $\alpha$-helices (known as TM37 and TM38) nearest to the protein's carboxyl terminus (Fig. 1).

For each arm, the structures of the three repeating units (which consist of TM25 to
TM36) nearest the pore were obtained at higher resolution than the next three units (which consist of TM13 to TM24). Each arm bends at the junction between these two trios of repeating units. Viewed from the extracellular side of the channel, the bend angle is about $100^{\circ}$, giving the channel the appearance of a clockwise spiral. When the trimer is viewed from the side, the bend angle is about $140^{\circ}$, so that the channel looks like an inverted dome. Analyses of the amino-acid sequence of mPiezol reported in the three new studies suggest that three additional repeat units (which consist of TM1 to TM12) form from the amino-terminal sequences of the protein and comprise the tip of each arm. However, these distal repeat units could not be resolved in the cryo-EM structures, perhaps because they are too flexible.

There are several notable structural features near the centre of the channel. On the extracellular side, there is a large cap on top of the central pore. On the cytoplasmic side, an $\alpha$-helix acts as a long 'beam' that connects TM28 to the central pore region. This beam might be involved in the mechanism by which force opens and closes the pore (the

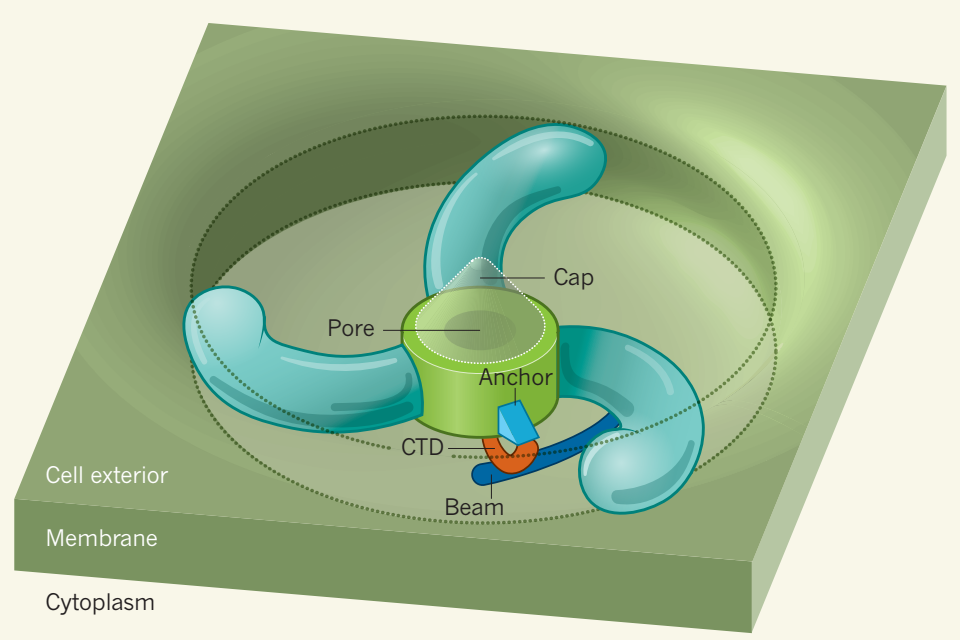

Figure 1 | Cartoon of the mechanosensitive mPiezol channel. Three papers ${ }^{2-4}$ report the structure of the mouse Piezo1 channel (mPiezo1), a membrane protein that responds to mechanical stimuli by opening its channel, thus allowing external ions to pass through the cell membrane into the cytoplasm. The channel forms from three mPiezol proteins, and consists of three bent arms surrounding a central pore, which has an extracellular cap. The cytoplasmic carboxy-terminal domain (CTD) of each mPiezol molecule extends from the pore to contact an anchor domain and a 'beam' that connects the arm to the pore. For simplicity, only one CTD, anchor and beam are shown. Guo and MacKinnon ${ }^{4}$ propose that the curvature of the arms induces deformation of the cell membrane; the membrane would fill the regions between the arms, but is not shown here for simplicity. 